\title{
Direct fabrication of nanowires in an electron microscope
}

\author{
N. Silvis-Cividjian, C. W. Hagen, ${ }^{\text {a) }}$ and P. Kruit \\ Delft University of Technology, Faculty of Applied Sciences, Lorentzweg 1, 2628 CJ Delft, The Netherlands
}

M. A. J. v.d. Stam and H. B. Groen

FEI Company, Electron Optics Product Division, PO Box 80066, 5600 KA Eindhoven, The Netherlands

(Received 6 December 2002; accepted 21 March 2003)

\begin{abstract}
Electron-beam-induced deposition (EBID) is a potentially fast and resistless deposition technique which might overcome the fundamental resolution limits of conventional electron-beam lithography. We advance the understanding of the EBID process by simulating the structure growth. The merit of our model is that it explains the shapes of structures grown by EBID quantitatively. It also predicts the possibility to directly fabricate structures with lateral sizes smaller than $10 \mathrm{~nm}$ and points out the ideal conditions to achieve this goal. We verify these predictions by fabricating sub-10-nm lines and dots in a state-of-the-art scanning transmission electron microscope. (C) 2003 American Institute of Physics. [DOI: 10.1063/1.1575506]
\end{abstract}

Energetic beams of photons, ions, and electrons are currently in use for fabrication of submicron devices for such diverse applications as microelectronics, nanophysics, and molecular biology. Among these, the focused electron beam fabricates the smallest features. The conventional electronbeam-induced lithography, based on polymethylmethacrylate resist has reached its fundamental resolution limits, situated around $10 \mathrm{~nm}$, as dictated by the interaction range of electrons with the resist, by the molecular size, and by the resist development mechanism. To fabricate even smaller structures, we investigate a resistless technique, called electronbeam-induced deposition (EBID), which might overcome the present resolution limitation problem.

Originally EBID was well known as contamination growth in electron microscopy. Broers et al. ${ }^{1}$ were the first to use contamination grown patterns as an etching mask to define 8-nm-wide metal lines. Only in the last decade has EBID gained more importance as a tool for additive lithography, ${ }^{2}$ practiced mainly in scanning electron microscopes (SEM). The principle of EBID is illustrated in Fig. 1 and can be described briefly as follows. In a high vacuum chamber, an electron beam is focused on a substrate surface on which precursor gas molecules, containing the element to be deposited (organometallic compound or hydrocarbon), are adsorbed. As a result of complex beam-induced surface reactions, the precursor molecules adsorbed in and near to the irradiated area, dissociate into nonvolatile (the deposit) and volatile fragments (to be pumped away). The advantage of EBID over conventional lithography methods is that two-, and even three-dimensional (3D), ${ }^{2-4}$ structures are patterned and deposited simultaneously, making it a fast, one-step technique.

The theoretical understanding of EBID is rather poor. Until now, there has not been a proper explanation for the fact that the smallest structures fabricated with EBID are typically $15-20$-nm wide, even though electron optical instruments, like SEMs and scanning transmission electron microscopes (STEMs), with much smaller probe sizes were

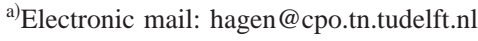

used. ${ }^{5-8}$ We improve the understanding by modeling the material growth under electron-beam irradiation. The merit of our model is not only that it explains the shapes of structures grown by EBID, but it also predicts the possibility to directly fabricate structures with lateral sizes smaller than $10 \mathrm{~nm}$ and points out the ideal conditions to achieve this goal. We show that these ideal conditions can be found in a state-of-the-art STEM, normally used as an analytical instrument.

We model the growth of a single dot, deposited by a focused electron beam, as follows. We follow each primary electron $(\mathrm{PE})$, and when it hits the substrate surface it is given an energy dependent probability to deposit material. Besides the primary electrons, the secondary electrons (SE) emitted from the target material, with energies ranging from 0 to $50 \mathrm{eV}$, play a very important role in the deposition, because the cross section for electron impact dissociation of gas molecules peaks at these low energies. In fact, in the case of high-energy PE beams, as in a SEM or STEM, the role of the PE in most cases can be neglected. The SE emitted from a flat substrate area larger than the beam diameter, may dissociate the adsorbed precursor molecules and a dot will start to grow. Its initial diameter is determined by the exit area of $\mathrm{SE}$ on the substrate surface. Although this scenario has been

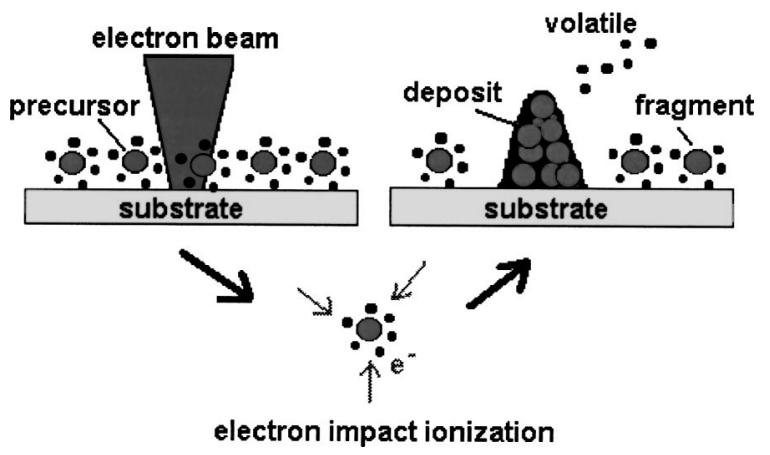

FIG. 1. Illustration of the EBID process. A precursor gas is continuously supplied to the substrate. An electron beam is focused on the substrate. The electrons are dissociating the adsorbed molecules and a deposit is formed on the substrate. The volatile components are evacuated from the deposition chamber. 


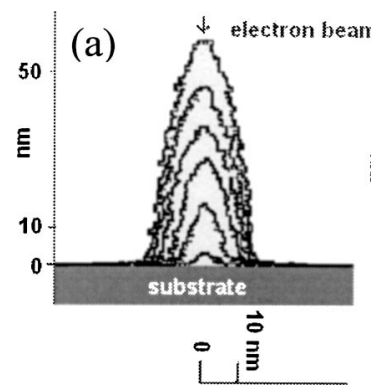

(b)

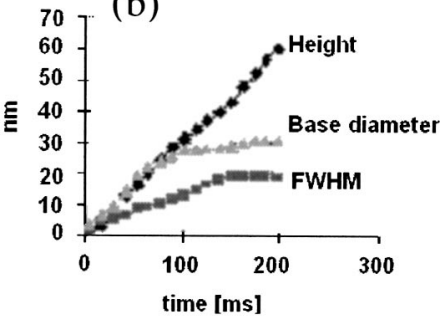

FIG. 2. A sequence of simulated profiles for a single carbon dot $\left(\mathrm{C}_{2} \mathrm{H}_{5}\right.$ precursor) deposited on a $10-\mathrm{nm}$ thin carbon foil by a $200-\mathrm{keV}$, zerodiameter electron beam, at normal incidence. (a) Cross-sectional profiles, only the profiles obtained at 18, 54, 90, 126, 162, and $198 \mathrm{~ms}$ after the deposition was started are shown. (b) The time evolution of the dot geometry: the dot height, the dot diameter measured at the half of its maximum height, and the dot diameter measured at the base are plotted. The saturation of the diameter is clearly observed.

brought up by many authors as an explanation for the growth of structures broader than the primary beam size, we have shown, using Monte Carlo simulations, that experimentally observed structure sizes cannot be explained this way. ${ }^{9,10}$ Therefore, we extend the model by including scattering in the growing structure as well. Weber ${ }^{11}$ also simulated electron scattering in tip-shaped specimen, and determined the electron range and the energy loss in the specimen, the shape of which remained static. However, we are interested in the effect of scattering in the continuously growing tip, that is, a dynamic tip shape. While the tip-like structure grows in vertical direction, the PE entering the apex of the tip may scatter in the tip, generating SE that can exit the tip from its side walls. These SE will dissociate the precursor molecules adsorbed on the tip flanks and thus will contribute to a lateral broadening. Saturation will occur when the SE are no longer able to exit the side flanks, as determined by the escape depth of the SE in the deposit $(5-15 \mathrm{~nm}) .{ }^{12}$

We developed a computer program, based on a twodimensional cellular automata method, to simulate the tip growth by SE only. ${ }^{13}$ During the simulation, the shape evolution of the dot is registered, and a typical result is presented in Fig. 2(a). A sequence of carbon dot profiles is shown, registered at equal time intervals, deposited on a $10-\mathrm{nm}$ carbon foil by a $200-\mathrm{keV}$, zero-beam-diameter electron beam, at normal incidence. We used $\mathrm{C}_{2} \mathrm{H}_{5}$ as a precursor gas because its dissociation cross section is known. Initially very small structures are obtained $(2-3 \mathrm{~nm})$, and as time proceeds, the width of the structure saturates $(20-30 \mathrm{~nm})$ while the height still increases. This is clearly seen in Fig. 2(b), where the diameter of the base of the tip, the full width at half-maximum (FWHM) of the tip, and the height of the tip are plotted versus time (the scale here is determined only by the $5 \mathrm{pA} \mathrm{PE}$ current). The simulated structure evolution is in good agreement with experimental observations from others. $^{5-8}$

Encouraged by the predictions of the simulation model, we performed carbon deposition experiments in a Tecnai $\mathrm{G}^{2}$ F20 U-TWIN STEM with a very stable and well-controlled electron-beam diameter smaller than $0.2 \mathrm{~nm} .{ }^{14}$ The substrate used was an unclean $10-\mathrm{nm}$ thin carbon foil that provided by itself sufficient contaminating hydrocarbons. In very thin substrates, PE scattering is low, resulting in a small surface Downloaded 17 Aug 2010 to 131.180.130.114. Redistribution subject to A (a)

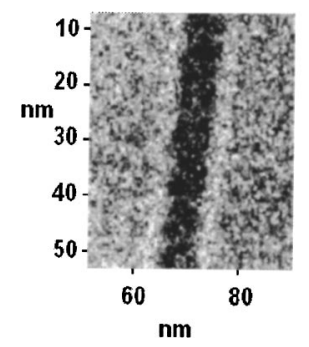

(b)

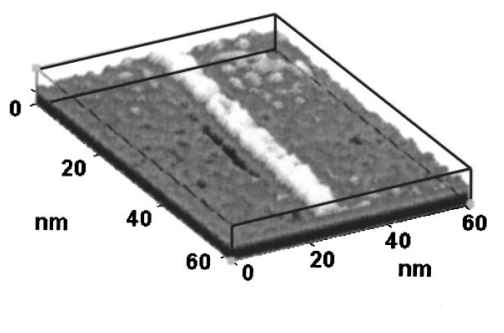

FIG. 3. Images of a line directly deposited on a thin carbon film in a STEM. (a) TEM micrograph and (b) a 3D intensity plot of the TEM image. The estimated average base width of the line is $8.3 \mathrm{~nm}$ and the FWHM is $6.5 \mathrm{~nm}$. The beam probe size was $1 \mathrm{~nm}$, probe current 5 pA, acceleration voltage 200 $\mathrm{keV}$, target thickness $10 \mathrm{~nm}$, scan length $1.28 \mu \mathrm{m}$, and exposure time $10 \mathrm{~s}$.

area from which the SE are emitted. In Fig. 3, a continuous wire is shown as obtained by scanning a $1-\mathrm{nm}$ probe, with 5 -pA current, twenty times along a $1.28-\mu \mathrm{m}$ long line with a repetition rate of $2 \mathrm{~Hz}$. Assuming a linear imaging model, a simple estimate of the FWHM is $6.5 \mathrm{~nm}$. The exposure dose, defined as the charge needed to fill a unit area with $6.5-\mathrm{nm}$ wide lines, is then $6010 \mathrm{C} / \mathrm{m}^{2} .{ }^{15}$

Five linear arrays of dots are shown in Fig. 4(a): three clearly visible arrays exposed with a dose of $10^{-13} \mathrm{C} / \mathrm{dot}$ and two less-visible arrays, in between the three, exposed with approximately $2 \times 10^{-14} \mathrm{C} /$ dot. The latter arrays are more clearly seen in the integrated cross-sectional plot across the arrays in Fig. 4(b), and have an estimated average dot diameter below $2 \mathrm{~nm}$.

Figure 5(a) shows a high-angle-annular-dark-field (HAADF) image of four sub-10-nm lines, written with a 0.2 -nm probe. From these images a more accurate line width can be obtained, as it is generally accepted that the HAADF contrast is proportional to the thickness. In Fig. 5(b), the integrated cross-sectional profile across the lines shows that the lines have an average base width of $9.5 \mathrm{~nm}$ and a FWHM of $4.3 \mathrm{~nm}$.

These structure sizes are about an order of magnitude smaller than the best results obtained by EBID performed in a SEM, and comparable or even smaller than the smallest features fabricated occasionally in scanning probe microscopes (SPM). ${ }^{16,17}$ In addition to that, EBID in a STEM has clear advantages over SPM-based techniques in that its probe

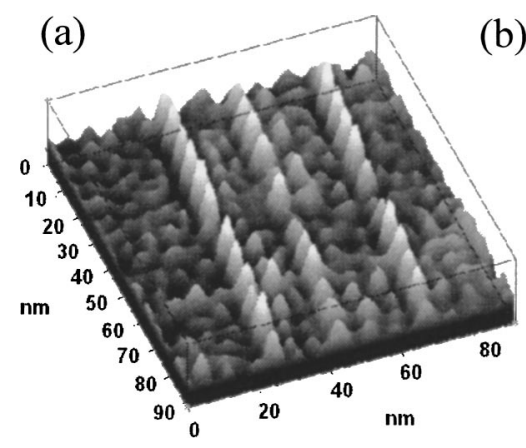

(b)

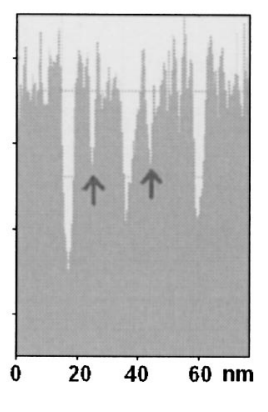

FIG. 4. Five arrays of dots deposited on a thin carbon film. (a) A 3D intensity plot and (b) an integrated cross-sectional plot across the parallel dot arrays. The arrows point to the smallest fabricated arrays of dots, with an estimated average dot diameter below $2 \mathrm{~nm}$. The beam probe size was $1 \mathrm{~nm}$, the three wider arrays were exposed at $10^{-13} \mathrm{C} / \mathrm{dot}$, and the two narrow ones at approximately $2 \times 10^{-14} \mathrm{C} /$ dot. 
(a)

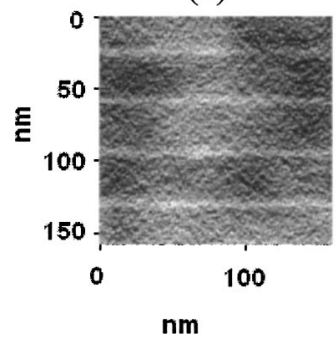

FIG. 5. A deposited pattern of four parallel continuous lines. (a) A HAADF image and (b) an integrated cross-sectional profile across the lines. The average base width of the lines is $9.5 \mathrm{~nm}$ and the average FWHM is $4.3 \mathrm{~nm}$. The probe size was $0.2 \mathrm{~nm}$.

size is known and adjustable, and it allows large area patterning at a much higher throughput.

In summary, a much better understanding of the EBID process was achieved from a detailed simulation of the structure growth. In the very first stage of the structure growth, the lateral structure size is determined by the secondary electrons generated in the substrate by the primary electrons, but at a later stage, the secondary electrons generated in the structure itself cause a lateral structure broadening which saturates in time. We have demonstrated that sub-10-nm structures can be easily fabricated in a state-of-the art STEM. The EBID-STEM technique may become very useful for the fabrication of all kinds of nanoscale devices. Sub-10-nm narrow lines and dots can be used to fabricate quantum devices, can serve as masks for metal deposition, as nanocontacts to measure the properties of molecules, proteins, and nanocrystals, or can fabricate critical size elements for laboratory-onchip technology and nanofluidics.
This work is part of the research program of the "Stichting voor Fundamenteel Onderzoek der Materie" (FOM), which is financially supported by the "Nederlandse Organisatie voor Wetenschappelijk Onderzoek" (NWO).

${ }^{1}$ A. N. Broers, W. W. Molzen, J. J. Cuomo, and N. D. Wittels, Appl. Phys. Lett. 29, 596 (1976).

${ }^{2}$ H. W. P. Koops, J. Kretz, M. Rudolph, M. Weber, G. Dahm, and K. L. Lee, Jpn. J. Appl. Phys. 33, 7099 (1994).

${ }^{3}$ H. W. P. Koops, E. Dobisz, and J. Urban, J. Vac. Sci. Technol. B 15, 1369 (1997).

${ }^{4}$ C. Schoessler and H. W. P. Koops, J. Vac. Sci. Technol. B 16, 862 (1998).

${ }^{5}$ K. T. Kohlmann-von Platen, J. Chlebek, M. Weiss, K. Reimer, H. Oertel, and W. H. Brünger, J. Vac. Sci. Technol. B 11, 2219 (1993).

${ }^{6}$ M. Komuro and H. Hiroshima, Microelectron. Eng. 35, 273 (1997).

${ }^{7}$ K. I. Schiffmann, Nanotechnology 4, 163 (1993).

${ }^{8}$ U. Hübner, R. Plontke, M. Blume, A. Reinhardt, and H. W. P. Koops, Microelectron. Eng. 57-58, 953 (2001).

${ }^{9}$ N. Silvis-Cividjian, C. W. Hagen, L. H. A. Leunissen, and P. Kruit, Microelectron. Eng. 61-62, 693 (2002).

${ }^{10}$ N. Silvis-Cividjian, Ph.D. thesis, DUP Science, Delft, 2002.

${ }^{11}$ M. Weber, J. Phys. D 27, 1363 (1994).

${ }^{12}$ L. Reimer, Scanning Electron microscopy (Springer, Berlin, 1990).

${ }^{13}$ This profile simulator will be described in a separate article.

${ }^{14}$ E. D. Boyes, J. Ringnalda, M. A. J. van der Stam, T. F. Fliervoet, and E. A. van Cappellen, Microsc. Microanal. 7, Suppl. 2, 232 (2001).

${ }^{15}$ It is noted here that this value is only three times larger than the statistical shot-noise limited dose ( $10^{4}$ electrons in a $1-\mathrm{nm}$ pixel), needed to write nanometer lines in resist-based electron-beam lithography.

${ }^{16}$ X.-D. Wang, S. Rubel, U. Purbach, and A. L. de Lozanne, Proc. SPIE 3009, 2 (1997).

${ }^{17}$ P. D. Szkutnik, A. Piednoir, A. Ronda, F. Marchi, D. Tonneau, H. Dallaporta, and M. Hanbücken, Appl. Surf. Sci. 164, 169 (2000). 